\title{
0 EFEITO DAS BEBIDAS ALCOÓLICAS PODE SER AFETADO PELA COMBINAÇÃO COM BEBIDAS ENERGÉTICAS? UM ESTUDO COM USUÁRIOS
}

Sionaldo Eduardo Ferreira, Marco Túlio de Mello, Maria Lucia Oliveira de Souza Formigoni*

Trabalho realizado no Departamento de Psicobiologia - UNIFESP, São Paulo, SP.

RESUMO - OBJETIVo. Avaliar em uma amostra de critério, o padrão de uso de bebidas energéticas, isoladamente e em associação com bebidas alcoólicas.

Métodos. Cento e trinta e seis voluntários (idade $24 \pm 6$ anos) que relataram ao menos um uso anterior de bebidas energéticas foram submetidos a uma entrevista padronizada sobre hábitos de consumo de bebidas energéticas e alcoólicas.

Resultados. A maioria da amostra relatou usar bebidas energéticas tanto isoladamente $(79 \%)$ como em combinação com bebidas alcoólicas $(76 \%)$, neste caso preferencialmente com uísque $(90 \%)$, vodka (37\%) ou cerveja (13\%). Após a ingestão isolada de bebidas energéticas, $61 \%$ relataram não sentir nenhum efeito, $10 \%$ mencionaram aumento da alegria, $9 \%$ euforia, $9 \%$ insônia, $7 \%$ desinibição e $24 \%$ aumento do vigor físico. Dos que relataram uso combinado com álcool, $14 \%$ relataram não sentir alteração dos efeitos do álcool, mas $38 \%$ reportaram aumento de alegria, euforia (30\%), insônia (II\%), desinibição (27\%) e do vigor físico (24\%). Observou-se grande variabilidade no número de usos de bebidas energéticas na vida $(14 \pm 16)$, mas certa regularidade na quantidade ingerida por ocasião (I,5 $\pm 0,7$ latas).

Conclusões. Os dados sugerem que os efeitos das bebidas energéticas são bastante variáveis, dependendo provavelmente da dose ingerida e da sensibilidade individual. Alguns relatos sugerem que há interação com o álcool, expressa pelo aumento dos efeitos excitatórios ou redução de seus efeitos depressores. São discutidos possíveis mecanismos farmacológicos subjacentes a esta combinação.

Unitermos: Bebidas energéticas. Bebidas alcoólicas. Cafeína. Taurina. Padrão de uso e interação.

\section{INTRODUÇÃo}

O consumo de bebidas alcoólicas é bastante antigo e de alta prevalência na população mundial. Em geral, quando ingeridas em baixa quantidade e freqüência, não geram problemas físicos e/ou psíquicos, sendo desta maneira consumidas pela maioria das pessoas. No entanto, o consumo abusivo de bebidas alcoólicas é bastante prejudicial, sendo a dependência de álcool uma das principais causas de problemas físicos e psíquicos atualmente.

Vários fatores modulam as respostas fisiológicas e comportamentais ao álcool, entre eles: características genéticas, estado geral de saúde, ambiente de uso (setting), dose e tempo de ingestão ${ }^{1,2,3}$.

Ao longo dos anos, diversas substâncias foram utilizadas com o intuito de amenizar os efeitos da intoxicação alcoólica, porém sem grande sucesso. Recentemente, observou-se

\begin{tabular}{c}
\hline *Correspondência: \\
R. Botucatu, $862-11^{\circ}$ Andar \\
CEP: $04.023-062$ - Vila Clementino - São Paulo - SP
\end{tabular}

a rápida popularização da ingestão de bebidas alcoólicas, principalmente destiladas, em combinação com bebidas energéticas" à base de carboidratos, taurina e cafeína. A comercialização destas bebidas é regulamentada pela Secretaria de Vigilância Sanitária do Ministério da Saúde.

Relatos populares sobre o aumento dos efeitos excitatórios do álcool e/ou de diminuição da intensidade dos seus efeitos depressores têm contribuído para a propagação do uso desta mistura. Porém, ainda não existem evidências científicas se as bebidas energéticas possuem de fato algum efeito antagonista dos efeitos depressores do álcool. Embora diversos relatos a respeito do uso combinado de bebidas energéticas e álcool tenham sido veiculados, a maior parte das informações não são cientificamente embasadas, sendo necessário estudos sobre o tema.

Especula-se que algumas substâncias presentes na composição das bebidas energéticas interfiram no metabolismo e/ou nas ações farmacológicas do álcool. Estudos com animais de laboratório têm demonstrado interações farmacológicas entre o álcool e a taurina, um dos componentes das bebidas energéticas ${ }^{4,5,6}$.

Trabalhos prévios demonstraram que a administração de bebida energética reverteu alguns dos efeitos depressores na atividade locomotora de camundongos induzidos por $2,5 \mathrm{~g} / \mathrm{kg}_{\mathrm{g}}$ de álcoo ${ }^{7,8}$. Entretanto, em seres humanos, há poucos estudos sobre os efeitos da ingestão de bebidas energéticas, especialmente em combinação com bebidas alcoólicas ${ }^{9-13}$.

Dado o reduzido número de trabalhos sobre o tema, este estudo teve por objetivo avaliar o padrão de uso das bebidas energéticas em uma amostra de critério formada por usuários destas bebidas.

\section{Métodos}

Amostra: Foi utilizada uma amostra de critério, composta por 136 voluntários, de ambos os sexos, com idade entre 14 e 42 anos, que relataram ao menos um uso de bebidas energéticas na vida. 


\section{Anexo I - Questionário sobre o padrão de uso de bebidas energéticas}

- Este questionário visa o conhecimento do Padrão de Uso de Bebidas Energéticas pela população em geral, por isso é importante que você responda corretamente a todas as perguntas que lhe serão feitas.

- Nenhum dosvoluntários desta pesquisaseráidentificado, sendo os dados aquiapontados de uso exclusivo parafins cientificos.

l) Idade:............anos Sexo: (a) M (b) F Altura:...............m Peso:..............g

2) Você já fez uso de Bebida Energética? (a) Sim (b) Não.

3) Se sim, quantas vezes aproximadamente?......................vezes

4) Normalmente, quantas latas consome?.......................latas

5) Você já usou bebidas energéticas junto com bebidas alcoólicas?
(a) Sim (b) Não. Se sim, com qual(is)? (a) Cerveja
(b) Vodka
(f) Outras.
$\begin{array}{ll}\text { (c) Uísque (d) Cachaça } & \text { (e) Tequila }\end{array}$

6A)Quais os efeitos que freqüentemente você sente depois de ingerir energéticos com álcool?

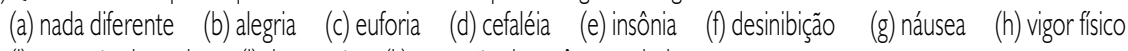

(i) sensaçãa de poder (j) depressão (k) sensação de estômago cheio.

6B)Quais os efeitos que freqüentemente você sente depois de ingerir energéticos sem álcool?
(a) nada diferente
(b) alegria (c) euforia
(d) cefaléía
(e) insônia (f) desinibiç̧ão
(g) náusea

(i) sensaçãa de poder (j) depressão (k) sensação de estômago cheio.

7) Em relação à primeira vez que ingeriu bebida energética, quando você ingere uma lata, os efeitos são:

(a) menores (b) maiores (c) continuam os mesmos (d) variáveis.

8) Em que ocasiöes você faz uso de bebida energética?
(a) atividade física
(b) trabalho
(c) dirigir veículos
(d) casas noturnas
(e) festas
(f) outras:.

9) Com que objetivo?

10) Espaço para comentários sobre as bebidas energéticas..

\begin{tabular}{lcc}
\hline Tabela I - Padrão de ingestão de bebidas energéticas: isoladamente ou em combinação com bebidas alcoólicas \\
\hline Ingestão isolada & & $79 \%$ \\
Número de latas por ocasião de uso & & $1,5 \pm 0,7$ \\
Número de usos na vida & $14,4 \pm 16,3$ \\
Intensidade dos efeitos em relação & menor & $10 \%$ \\
ao primeiro uso & maior & $4 \%$ \\
& igual & 71 \\
& varível & 5 \\
& só usou uma vez & 9 \\
Local de uso & academias & 20 \\
& trabalho & 4 \\
& direção de vé́culos & 3 \\
& casas noturnas & 71 \\
Ingestão com bebidas alcoólicas & festas & 50 \\
Bebida accólica de preferência & & 76 \\
para uso combinado & uísque & 90 \\
& vodka & 37 \\
& cerveja & 13 \\
& outras & 11 \\
\hline
\end{tabular}

Procedimentos: Os voluntários foram convidados pelos pesquisadores através de abordagem direta na entrada de casas noturnas, academias de ginástica e na Universidade Federal de São Paulo, na cidade de São Paulo, SP. Após esclarecimento sobre os objetivos do projeto e garantia de sigilo e anonimato, foi aplicada uma entrevista padronizada que incluía dados demográficos (idade e sexo), índices corporais (peso e altura) e questões sobre o uso de bebidas alcoólicas e energéticas (Anexo I). Apenas os indivíduos que haviam feito uso anterior de bebidas energéticas e concordaram em responder às perguntas da entrevista foram admitidos como voluntários. O estudo foi aprovado pelo Comitê de Ética em Pesquisa da UNIFESP (394/00).

\section{Resultados}

A amostra incluiu proporção semelhante de homens (56\%) e mulheres (44\%), sendo a idade média dos entrevistados de $24 \pm 6$ anos. A Tabela I apresenta as principais características do padrão de uso das bebidas energéticas. Observou-se grande variabilidade na frequiência de uso de bebidas energéticas, observamos desde uso único ( $9 \%$ dos casos) a até 100 usos na vida conforme relatado ( $14 \pm$ 16 usos MD $\pm D P$ ). Entretanto, observou-se certa regularidade na quantidade consumida por ocasião de uso, variando entre uma e três latas por ocasião ( $1,5 \pm 0,7$ latas $M D \pm D P)$.

A maioria dos entrevistados relatou fazer uso de bebidas energéticas em casas noturnas (7। \%) e festas (50\%), mas também foi relatado uso durante a realização de atividades físicas em academias (20\%), durante o trabalho (4\%) e antes de dirigir veículos (3\%).

Quanto às razões de uso, cerca de 30\% da amostra comentou usar uísque com bebida energética para se manterem estimulados a noite toda. Reduzindo assim, com a combinação, alguns dos efeitos depressores do álcool. Alguns entrevistados relataram usar a bebida energética para diluir e melhorar o sabor das bebidas alcoólicas, considerado pouco agradável. Ocorreram relatos de uso de bebidas energéticas como opção alternativa a outras bebidas, às vezes em substituição a bebidas alcoólicas ou refrigerante, por exemplo.

A maioria dos entrevistados (76\%) relatou fazer uso de bebidas energéticas de forma combinada com bebidas alcoólicas, sendo que, neste caso, 91\% utilizavam bebidas destiladas. Preferencialmente, as bebidas energéticas eram ingeridas em combinação com uísque (90\%), vodka (37\%) e cerveja (।3\%). O relato de uso de bebida energética pura também foi freqüente (79\%).

Considerando-se toda a amostra, apenas 10\% relataram tolerância (redução dos efeitos) e $4 \%$ aumento dos efeitos em relação ao primeiro uso. De modo geral, os usuários relataram efeitos agradáveis, sendo baixa a 


\begin{tabular}{lcc}
\hline \multicolumn{2}{l}{ Tabela 2-Efeitos percebidos após a ingestão de bebida energética pura e em combinação com bebidas alcoólicas } \\
\hline Efeitos percebidos (\%) & Bebida energética pura & Bebida energética com álcool \\
Nada differente & 61 & 14 \\
Alegria & 10 & 38 \\
Euforia & 9 & 30 \\
Cefaléia & 3 & 2 \\
Insônia & 9 & 11 \\
Desinibição & 7 & 27 \\
Náusea & 1 & 0 \\
Vigorfísico & 24 & 24 \\
Sensação de poder & 1 & 5 \\
Depressão & 0 & 0 \\
Plenitude gástrica & 6 & 4 \\
\hline
\end{tabular}

ocorrência de efeitos desagradáveis (12\%). Nestes casos, os efeitos mais relatados foram insônia (7\%), sensação de plenitude gástrica (3,5\%) e cefaléia (I,5\%). Não foram detectadas diferenças entre o uso isolado e combinado com bebidas alcoólicas em relação aos efeitos desagradáveis.

Em relação ao uso de bebidas energéticas, a maioria relatou não observar efeitos significativos após sua ingestão isolada (6/\%). Entretanto, ao mencionar os efeitos percebidos após a ingestão de bebidas energéticas em combinação com bebidas alcoólicas, 86\% da amostra relatou alguma alteração, como aumento da alegria (38\%), da euforia (30\%), da desinibição (27\%) e do vigor físico (24\%). A Tabela 2 apresenta os principais efeitos percebidos após a ingestão de bebidas energéticas isoladamente e após combinação com bebidas alcoólicas.

\section{Discussão}

São poucos os estudos sobre os efeitos da ingestão de bebidas energéticas, sendo este, ao nosso conhecimento, o primeiro sobre seu padrão de uso no Brasil.

Os resultados obtidos indicam que apesar das recomendações inseridas no rótulo do produto, a respeito de se evitar sua mistura com álcool, esta prática é bastante comum, especialmente entre freqüentadores de casas noturnas. Observou-se grande variabilidade individual quanto aos efeitos relatados.

Uma importante questão sobre o uso de bebidas energéticas é a possível alteração do padrão de uso de bebidas alcoólicas, especialmente das destiladas. Grande parte da amostra relatou não ter por hábito ingerir uísque e/ou vodka, mas o fazer, e por vezes em grande quantidade, quando em combinação com bebidas energéticas. Isto sugere que a melhora no sabor, obtida pela mistura, poderia estimular a ingestão de maiores quantidades de bebidas destiladas.

Se a sensação de redução dos efeitos depressores do álcool não for acompanhada por redução do prejuízo na coordenação motora e no tempo de reação a estímulos, o indivíduo sob efeito da mistura pode superestimar sua capacidade de desempenhar atividades, aumentando assim o risco de se envolver em acidentes. Estudos com voluntários demonstraram que a ingestão de uma lata de bebida energética não reduziu os prejuízos provocados pela ingestão de álcool $(0,6$ e I,0 g/ $/ \mathrm{g})$ nas capacidades de atenção, tempo de reação visual e coordenação motora ${ }^{13}$.

Entre os poucos trabalhos encontrados na literatura a respeito da interação entre álcool e bebidas energéticas, destaca-se o de Riesselman et al. (1996) que sugeriram que usuários desta combinação poderiam fazer um juízo errôneo de suas capacidades e provocar acidentes com maior probabilidade do que somente após a ingestão de álcool.

Os dados obtidos até o momento indicam que as bebidas energéticas, especialmente aquelas que contém taurina e cafeína, vêm sendo utilizadas para potencializar os efeitos estimulantes do álcool. Resta identificar se este efeito é farmacologicamente explicável e quais os mecanismos envolvidos, ou se é apenas um efeito placebo.

Constam na literatura alguns trabalhos relatando os efeitos da ingestão de bebidas energéticas como melhora do desempenho psicomotor, redução do tempo de reação motora, aumento da concentração ou da memória imediata e aumento da sensação subjetiva de alerta ou vigor. Há também relatos sobre a melhora do estado de humor e do desempenho físico após a ingestão de Red Bull ${ }^{\otimes 9}, 10,11$. Nestes estudos, os autores atribuem os efeitos à ingestão do composto, mas como não testaram seus componentes isoladamente não puderam sugerir os prováveis mecanismos de ação.

No presente estudo, os relatos de diminuição do sono e de aumento da sensação de prazer ao se ingerir bebidas alcoólicas em combinação com energéticas sugerem que estas poderiam prolongar a duração dos efeitos excitatórios do álcool. Uma possibilidade de explicação para este efeito seria uma modulação da neurotransmissão gabaérgica exercida pela taurina. Sabe-se que o efeito depressor do álcool está associado a aumento da neurotransmissão mediada pelo GABA (ácido gama-amino-butírico). Desta forma, diminuindo a atividade gabaérgica, a taurina reduziria o efeito depressor do álcool ${ }^{14}$. É possível também que a redução do efeito depressor do álcool seja devida às ações estimulantes da cafeína no córtex cerebral. Alguns estudos demonstram que em determinadas doses a co-administração de cafeína reduz alguns dos efeitos depressores do álcool ${ }^{15,16}$. Entretanto, não há estudos sobre a administração conjunta de taurina, cafeína e álcool que permitam fortalecer alguma destas hipóteses.

A ampliação e diversificação desta amostra são fundamentais para o melhor conhecimento dos hábitos de uso e efeitos observados após a ingestão combinada de bebidas alcoólicas e energéticas, que popularizou-se e vem aumentando rapidamente, especialmente entre a população mais jovem.

Encontram-se em andamento estudos com voluntários e em animais de laboratório que têm por objetivo avaliar, de maneira sistematizada, os efeitos agudos e crônicos da ingestão de álcool com bebidas energéticas.

\section{SUMMARY}

Can energy drinks affect the EFFECTS OF ALCOHOLIC BEVERAGES? A STUDY WITH USERS

OBJECTNES. The pattern of use of energy drinks, alone or combined with alcoholic beverages, was evaluated in a criteria sample. 
Methods. 136 volunteers aged $24 \pm 6$ years, who had reported at least one previous use of energy drinks, answered a questionnaire on their pattern of use of energy drinks and alcoholic beverages.

RESULTS. Most of the sample (76\%) reported using energy drinks in combination with alcoholic beverages, preferably whisky (90\%), vodka (37\%) and beer (13\%). Most of the sample (79\%) also reported using energy drinks alone. In the latter case, $61 \%$ of them reported not feeling any effect, 10\% reported happiness, $9 \%$ euphoria, $9 \%$ insomnia, $7 \%$ uninhibited behavior and 24\% increase in physical vigor. Out of those who reported combined use with alcohol, $14 \%$ told not to feel different, 38\% increase happiness, 30\% euphoria, I1\% insomnia, 27\% uninhibited behavior and 24\% increase of physical vigor. High level of variability was observed in the number of lifetime uses of energy drinks (14 \pm 16). However, there was certain constancy in the number of cans ingested per occasion ( $1.5 \pm 0.7)$.

Conclusions. It was observed that the effects of energy drinks are variable, probably depending on the dose and individual sensitivity. Some reports suggest interaction with alcohol, expressed as an increase in the excitatory effects or reduction in the depressive effects. The possible pharmacological mechanisms underlying this interaction are discussed. [Rev Assoc Med Bras 2004; 50(I): 48-5I]

KEY wORDs: Energy drinks. Caffeine. Taurine. Pattern of use. Alcoholic beverages and interaction.

\section{Agradecimentos}

Os autores agradecem a participação de Marcio Vinícius Rossi, Renata Guedes Koyama e Rita Aurélia Boscolo Pereira na coleta de dados desta pesquisa. Esta pesquisa recebeu apoio financeiro da Associação Fundo de Incentivo à Psicofarmacologia (AFIP), do CNPq e da FAPESP (00/I 5043-7) e apoio estrutural do Centro de Estudos em Psicobiologia e Exercício (CEPE/UNIFESP).

\section{REFERÊNCIAS}

I. Charness ME, Simon RP, Greenberg. Ethanol and the Nervous System. N Engl J Med 1989; 32 I (7):442-54.

2. Kalant $H$. Current trends in biomedical research on alcohol. Alcohol Alcoholism Suppl 1987; (I): I- 12.

3. Valenzuela CF. Alcohol and neurotransmitter interactions. Alcohol Health Res W 1997; 2 I (2): I 44-8.

4. Aragon CMG, Trudeau LE, Amit Z. Effect of taurine on ethanol-induced changes in openfield locomotor activity. Psychopharmacology 1992; 107(2/3):337-40.

5. Dahchour A, Quertemont E, De Witte P. Taurine increases in the nucleus accumbens microdialysate after ethanol administration to naive and chronically alcholised rats. Brain Res 1996; 735(I):9-19.

6. Kerai MD, Waterfield CJ, Kenyon SH, Asker DS, Timbrell JA. Taurine: protective properties against ethanol-induced hepatic steatosis and peroxidation during chronic ethanol consumption in rats. Amino Acids 1998; I 5(I-2):53-76.

7. Ferreira SE, Quadros IMH, Mello MT, Formigoni MLOS. Atividade locomotora de camundongos após administração de etanol com bebidas energéticas. In: Reunião Anual da Federação das Sociedades de Biologia Experimental (FeSBE). Caxambú - MG, 200I. Resumos. p. 42
8. Quadros IMH, Takahashi S, Bianchi MF, Trindade AA, Ferreira SE, Souza-Formigoni MLO. A co-administração de bebida energética impede o efeito depressor do etanol sobre a atividade locomotora de camundongos. In: XVII Reunião Anual da Federação das Sociedades de Biologia Experimental (FeSBE). Salvador - BA, 2002. Resumos. p. 126.

9. Alford C, Cox H, Wescott R. The effects of red bull energy drink on human performance and mood. Amino Acids 200 I; 2 I (2): 139-50.

10. Horne JA, Reyner LA. Benefical effects of "energy drink" give sleepy drivers. Amino Acids 200 I; 20( I):83-9.

I I. Seidl R, Peyrl A, Nicham R, Hauser E. Taurine and caffeine-containing drink stimulates cognitive performance and well-being. Amino Acids 2000; 19(3-4):635-42.

12. Riesselman B, Rosenbaum F, Schneider V. Alcohol and energy drink can combined consumption of both beverages modify automobile driving? Blutalkohol 1996; 33:4

13. Ferreira SE. Estudo dos efeitos fisiológicos e psicológicos da ingestão combinada de álcool e bebidas energéticas (dissertação). São Paulo: Universidade Federal de São Paulo, Escola Paulista de Medicina; 2002.

14. Kuriyama K, Hashimoto T. Interrelationship between taurine and GABA. Adv Exp Med Biol 1998; 442:329-37.

15. Fudin R, Nicastro R. Can caffeine antagonize alcohol-induced performance decrements in humans? Percept Motor Skills 1988; 67(2):375-91.

16. Liguori A, Robinson JH. Caffeine antagonism of alcohol-induced driving impairment. Drug Alcohol Dependence 200 I; 63(2): I23-9.

Artigo recebido: 24/10/02 Aceito para publicação: 19/09/03 